\title{
Social restriction versus herd immunity policies in the early phase of the SARS-CoV-2 pandemic: A mathematical modelling study
}

Wiwat Chancharoenthana, ${ }^{1}$ Asada Leelahavanichkul, ${ }^{2,3}$ Sutatip Chinpraditsuk, ${ }^{4}$ Krit Pongpirul, ${ }^{5,6}$ Supitcha Kamolratanakul, ${ }^{1}$ Weerapong Phumratanaprapin, ${ }^{1}$ Polrat Wilairatana, ${ }^{1}$ Punnee Pitisuttithum ${ }^{1}$

\begin{abstract}
Background: Two main strategies to cope with the coronavirus disease 2019 (COVID-19) pandemic-lockdown (social restriction) and non-lockdown (herd immunity plan)-have been implemented in several countries.

Objective: This study aims to statistically compare the outcomes of the two strategies, represented by data from Thailand and Sweden, respectively.

Methods: Data for COVID-19 pandemic control from Thailand, representing social restriction, versus data from Sweden, representing the herd immunity plan, collected from January 13 to May 31, 2020, were analyzed by using the SIR (susceptible, infectious, recovered) model.

Results: The SIR model analysis demonstrated a beneficial effect of each model on the attenuation of the mortality rate, with lower mortality in social restriction and shorter overall pandemic duration in the herd immunity plan. However, the herd immunity plan demonstrated a higher mortality rate than social restriction (46.9\% versus $1.9 \%$ ) despite the later entry of the virus in Sweden. When the SIR model was used for predicting the COVID-19 status, Sweden was shown to likely end its COVID-19 epidemic earlier than Thailand (268 vs. 368 days). With the nonlinear estimation, at least one log difference between total confirmed cases versus active cases could be used as an indicator for relaxation of the lockdown policy in Thailand.
\end{abstract}

Conclusions: Both the social restriction and herd immunity plans are beneficial for COVID-19 pandemic control in terms of the amelioration of pandemic mortality. The cumulative number of total recovered cases might be a potential parameter that could be used for determining the policy direction for COVID-19 control.

Key words: COVID-19, control strategy, lockdown, mortality rate, SARS-CoV-2

From:

1 Department of Clinical Tropical Medicine,

Faculty of Tropical Medicine, Mahidol University, Bangkok, Thailand

Department of Microbiology, Faculty of Medicine,

Chulalongkorn University, Bangkok, Thailand

3 Translational Research in Inflammation and Immunology Research

Unit (TRIRU), Department of Microbiology, Faculty of Medicine,

Chulalongkorn University, Bangkok, Thailand

Hospital for Tropical Diseases, Faculty of Tropical Medicine,

Mahidol University, Bangkok, Thailand

Thailand Research Center for Health Services System (TRC-HS), Faculty of Medicine, Chulalongkorn University, Bangkok, Thailand

6 Department of International Health, Johns Hopkins Bloomberg School of Public Health, Baltimore, MD, USA

\section{Corresponding author:}

Wiwat Chancharoenthana

Department of Clinical Tropical Medicine,

Faculty of Tropical Medicine, Mahidol University

16/F Ratchanakarin Building 420/6 Rajvithi Rd., Ratchathewi,

Bangkok 10400, Thailand

E-mail: wiwat.cha@mahidol.ac.th

\section{Introduction}

Coronavirus disease 2019 (COVID-19) was first reported in Wuhan, China, and has since become a pandemic disease. The spreading pattern and transmission differ from those of SARS-CoV-1, as SARS-CoV-2 is shed from the upper respiratory tract, while SARS-CoV-1 replication occurs mainly in the lower respiratory tract. ${ }^{1}$ SARS-CoV-2 transmission occurs principally through respiratory droplets, so avoiding the 
droplets by '2-meter social (physical) distancing' is the main strategy to prevent the contagious transmission. The wearing of face masks and frequent handwashing are additional recommendations. Accordingly, many countries encourage social restriction at various intensity levels, ranging from stay-at-home advice to complete lockdown. Hong Kong is a good example of effective COVID-19 control via a lockdown policy, and this policy has been adopted by the Thai government. Although social distancing with the lockdown policy reduces the patient load and, therefore, the mortality rate of the COVID-19 pandemic, this approach is difficult in the long run, mostly due to the loss of financial security. In contrast, herd immunity is an epidemiological concept in which the presence of enough people with effective immunity to protect vulnerable people prevents the infection from spreading within the community. ${ }^{2}$

Thailand encountered its first COVID-19 case on January 12 , 2020. Since then, people have been urged to wear face masks, stay and work from home, and avoid group activities. Several recommendations regulated by the Thai government were also implemented to prevent nationwide spreading. Of note, the restriction law was first enforced by the Bangkok Metropolitan Administration (BMA) on March 22, 2020, when the incidence of COVID-19 cases in Thailand reached its maximum rate (188 cases within 24 hours). Shopping malls, markets, parks, schools, athletic centers, barbershops, night clubs, and other crowded places were closed, and group activities were prohibited. The Thai Emergency Decree on Public Administration in Emergency Situation due to the Outbreak of the Coronavirus 2019 (COVID-19) was announced on March 26, 2020. All airline services were stopped, and an effective 'curfew' was instated from 10 p.m. to 4 a.m. from April 3, 2020, onward. In Sweden, the herd immunity plan allowed normal daily life activity during the pandemic (Supplemental data).

Because i) COVID-19 transmission is similar to the process of diffusion into a crowd, ${ }^{3,4}$ ii) a prediction model for transmission analysis can be used to predict the trend of disease spreading, ${ }^{5}$ and iii) a clear indicator for choosing between these 2 policies is not available, we used model analysis to test the difference between the social restriction model (model A) vs. the herd immunity (with no social restriction) plan (model B). This information could be adopted to cope with the second wave of COVID-19 when it occurs.

\section{Material and Methods \\ Data Collection}

The study protocol has been submitted to the Ethics Committee of the Faculty of Tropical Medicine, Mahidol University, Thailand, with proof of exemption (MUTM-EXMPT 2020003). Daily Thai COVID-19 data were mainly obtained from the Department of Disease Control, Ministry of Public Health of Thailand, ${ }^{6}$ and the COVID-19 World Health Organization (WHO) Thailand Situation Reports. ${ }^{7}$ The retrieved data were systematically sorted from January 10, 2020, when awareness of surveillance of the novel coronavirus associated with pneumonia from travelers arrived in Thailand, to May 31, 2020.
Thailand (model A) has used 'the hammer and the dance' strategy since the first COVID-19 epidemic wave, including nationwide lockdown, business closure, stay-at-home measures, and physical distancing along with intense surveillance, isolation of infected individuals, and tracing and quarantining of their contacts. Meanwhile, Sweden opted for a herd immunity strategy (model B). The stringency index obtained from a publication was used to clarify the effect of social restriction by both countries, and 17 indicators of government responses were aggregated as policy scores for the individual countries. ${ }^{8}$ However, the original stringency index that used in the present study mainly inferred to the strictness of 'lockdown style' policies that primarily restrict people's behavior. Although the containment and health index, which represent the combination of lockdown restrictions and closures with measures such as testing policy and contact tracing, short-term investments in healthcare and investments in vaccine, seems affect to both strategy outcomes, these indices were not included owing to limited data as the early phase of the pandemic. Moreover, it should be noted that the stringency index score did not include financial aspects and must not be interpreted as a score of the appropriateness or effectiveness of a country's response to the COVID-19 pandemic. The COVID-19-related data of both countries were obtained from the World Health Organization (WHO) website and other reliable publicly available websites, including the Worldometer website, ${ }^{9}$ Coronatracker website, ${ }^{10}$ and Johns Hopkins Coronavirus Resource Center. ${ }^{11}$ All data were retrieved twice a day, in the morning and evening, because of the time zone difference. Then, data were compared and counter-checked among available resources for data validity. If the retrieved data showed inconsistency among those resources, the referenced data from the Department of Disease Control, Ministry of Public Health of Thailand, and the Worldometer website were prioritize set as the raw data for Thailand and Sweden, respectively. Indeed, we found more compatible data between the reported case of Thailand from the Department of Disease Control, Ministry of Public Health of Thailand, and the Worldometer website rather than the other resources.

\section{Data Analyses}

The retrieved data above were used in the infection model analysis. The infection model analysis used in the present study was a classic SIR (susceptible, infectious, recovered) analysis as follows: ${ }^{12}$

$$
\begin{aligned}
& \frac{d S_{t}}{d t}=-\beta S_{t} I_{t} \\
& \frac{d I_{t}}{d t}=\beta S_{t} I_{t}-\gamma I_{t} \\
& \frac{d R_{t}}{d t}=\gamma I_{t}
\end{aligned}
$$
tially.

where $N_{t}=S_{t}+I_{t}+R_{t}$, and $S=S_{0}, I=I_{0}$, and $R=0$, ini- 
The SIR parameters were derived from the data obtained from the equivalent published estimation data (i.e., $R_{0}, \gamma, \beta$ ) and then applied to the actual country data. $\beta$ indicates the probability of infection between the susceptible individuals and the latent cases, that is, $\beta$ also represents the effective contact rate; $\gamma$ represents the recovery rate, which is the reciprocal of the number of days in the treatment periods. $\gamma$ was assumed to be primarily a biological and fixed value over time in all countries. In this study, $\gamma=0.1$, as the average length of time a person is infectious is $1 / \gamma$, so a 10 -day duration for the treatment cycle was selected in our study. $R_{0}$ refers to the number of secondary cases generated by a typical infectious individual when the rest of the population is susceptible. $R_{0}$ $=\beta \times 1 / \gamma$ (or No. of infections from one sick person $=$ No. of lengthy contacts per day $\times$ No. of days contacts are infectious). This means that the changing $\beta$ is associated with social distancing. In other words, with a constant $\beta$, the number of people infected in the long run can be pinned down by $R_{0}$. As a result of social restriction in Thailand, the $R_{0}$ was $2.48,{ }^{13}$ while the herd immunity strategy for COVID-19 led to $R_{0}=$ 3.2 for Sweden. ${ }^{14}$ Accordingly, the effective contact rate $(\beta)$ was 0.248 and 0.32 for Thailand and Sweden, respectively. The transmission rate was 2.0, estimated from an average value of $R_{0}$ from various studies. ${ }^{15-20}$ The contact rates in the present study were $12.4 \%$ and $16 \%$ for Thailand and Sweden, respectively. In comparison, the contact rate in China was $9.1 \%$ and $15.4 \%$ from the epidemiological and transmission studies, ${ }^{16}$ with the reference age group of contacts being 50-59 years and 60-69 years, respectively.

The total population of Thailand and Sweden is 69.8 and 10.1 million, respectively. ${ }^{21}$ Although data from the cohort study of COVID-19 in Wuhan, China, ${ }^{17}$ demonstrated that the case mortality rate was very high in the early stage of the pandemic $(28.3 \%)$, the mortality rates in Thailand and Sweden as of May 31, 2020, were $1.89 \%$ and $46.9 \%$, respectively. ${ }^{9,11}$ We indicated that social restriction strategies could reduce reproduction or ' $R$ ' to values below one $(R<1) .^{22}$ The clinical outcome prediction for COVID-19 in Thailand and Sweden was analyzed based on epidemiological data from China, as $13.8 \%$ of the total predicted cases require hospitalization and $4.7 \%$ of the total predicted cases require intensive care facilities. ${ }^{23}$ To minimize bias in data fitting, cubic spline interpolation was used for curving the given data points. ${ }^{24}$ Statistical analyses were performed with STATA 13.0 (StataCorp LP, College Station, TX) whereas the SIR model and cubic spline interpolation were calculated and performed by using Excel spreadsheet and GraphPad Prism version 8.0 for Windows (GraphPad Software, San Diego, CA), respectively. Full details of the Excel spreadsheet have been described elsewhere. ${ }^{25} \mathrm{We}$ also further studied the parameters that determine the good outcomes following relaxation of social restriction in selected countries worldwide and validated the parameters with actual situation (good, fair, and needed urgent action) which have been classified previoulsy. ${ }^{26}$

\section{Results}

By May 31, 2020, COVID-19 had affected 213 countries and territories around the world. These included 6,259,250 confirmed cases along with 374,138 deaths and 2,843,986 recovered. ${ }^{911}$ Of note, the total number of confirmed cases of COVID-19 in Thailand (model A) was 3,081, with 61 active cases remaining in hospitals (Table 1 and Figure 1A).

Table 1. Reported coronavirus cases as of May 31, 2020.

\begin{tabular}{|c|c|c|c|}
\hline \multirow{2}{*}{ Parameters } & \multirow{2}{*}{ World } & \multicolumn{2}{|c|}{ Countries } \\
\hline & & Thailand & Sweden \\
\hline Total cases & $6,259,250$ & 3,081 & 37,542 \\
\hline New cases ${ }^{*}$ & $+108,768$ & +4 & +429 \\
\hline Total deaths & 374,138 & 57 & 4,395 \\
\hline New deaths* & $+3,189$ & 0 & 0 \\
\hline Total recovered & $2,843,986$ & 2,963 & 4,971 \\
\hline $\begin{array}{l}\text { Closed cases with had an } \\
\text { outcome }\end{array}$ & N/A & 3,020 & 9,366 \\
\hline $\begin{array}{l}\text { Recovery/discharged, } \\
\mathrm{n}(\%)\end{array}$ & N/A & $2,963(98.1)$ & $4,971(53.1)$ \\
\hline Deaths, n(\%) & N/A & 57 91.9) & $4,395(46.9)$ \\
\hline Active cases & $3,041,126$ & 61 & 28,176 \\
\hline Critical cases & 53,409 & 1 & 228 \\
\hline $\begin{array}{l}\text { Total cases/ } 1 \text { million } \\
\text { population }\end{array}$ & 803 & 44 & 3,719 \\
\hline $\begin{array}{l}\text { Deaths/1 million popu- } \\
\text { lation }\end{array}$ & 48.0 & 0.8 & 435 \\
\hline
\end{tabular}

* Compared with previous day (May 30, 2020).

$(+)$ indicates an increase in reported cases. N/A, data not available.

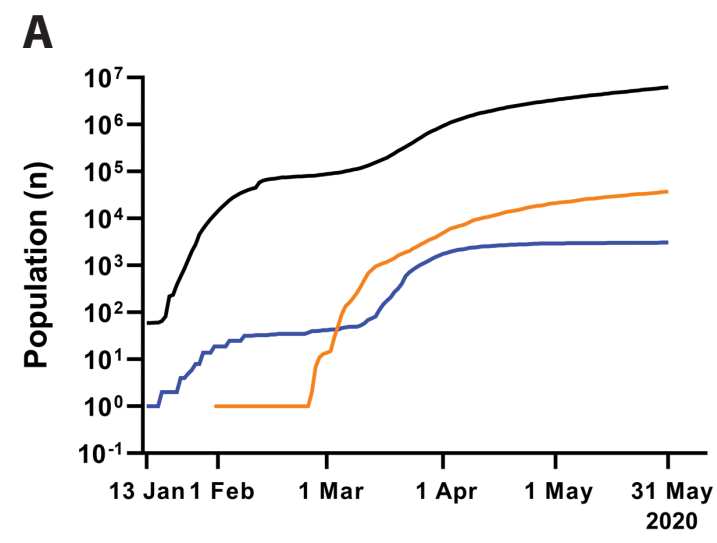

— World — Thailand (model A) — Sweden (model B)

Figure 1. Total confirmed COVID-19 cases and stringency index.

(A) The total confirmed COVID-19 cases in Thailand (model A) and Sweden (model B) were compared with the total cases of COVID-19 worldwide from January 13 until May 31, 2020. 
B
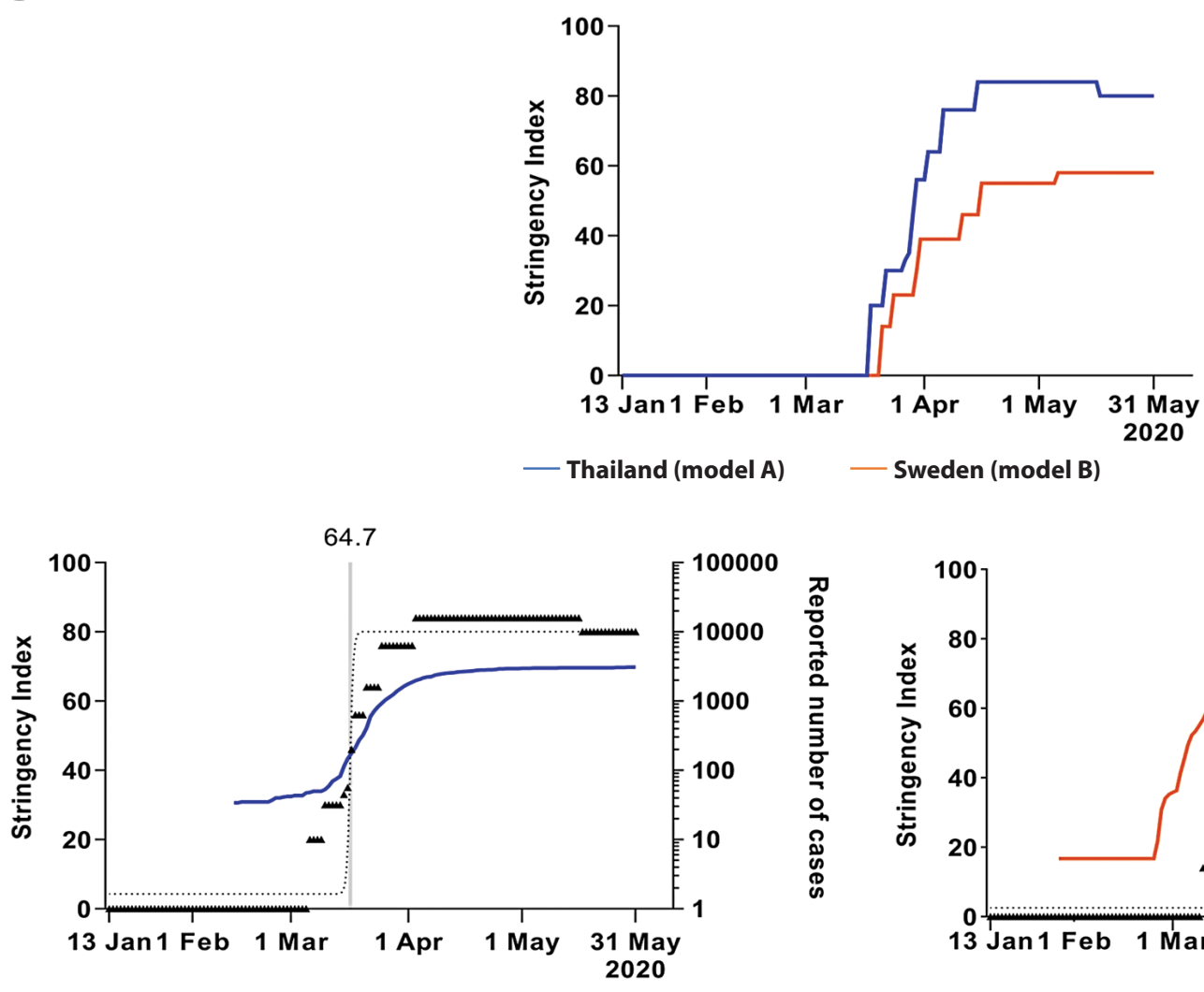

*. Stringency index - Reported number of case

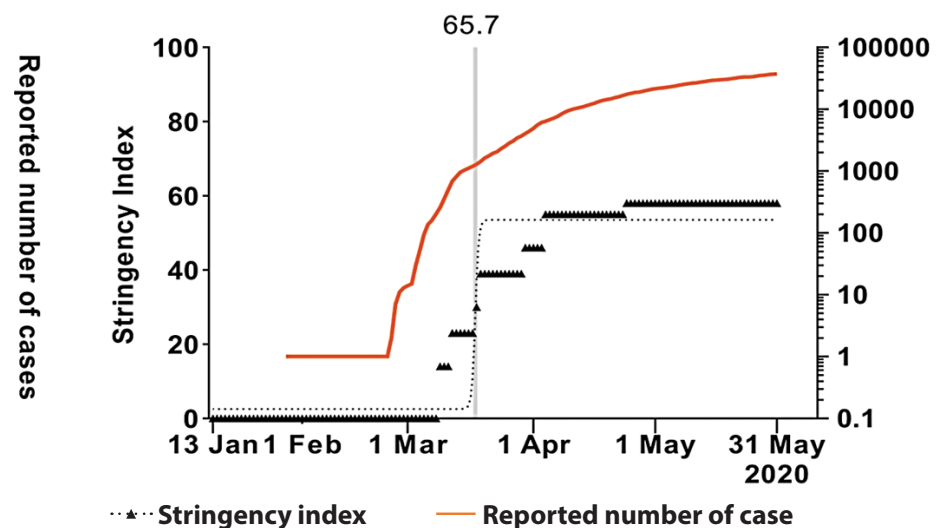

Figure 1. (Continued)

(B) Stringency index of each model during January13-May 31, 2020. Comparison of the stringency index pattern between models (upper panel). Thailand (middle panel) and Sweden (lower panel), the representatives of models A and B, respectively, showed comparable timing of government restriction policies in response to COVID-19 (64.7 vs 65.7 days, fitting by nonlinear approximation).

Meanwhile, Sweden (model B) reported 37,542 confirmed cases (equivalent to a crude rate of 3,719 cases per million population). Approximately 238,800 COVID-19 tests (or 23,657 tests per million population) were performed in Sweden, compared with 375,453 samples (or 5,380 tests per million population) of COVID-19 tests in Thailand (according to the data from 121 of total 142 COVID-19 RT-PCR laboratories).

A

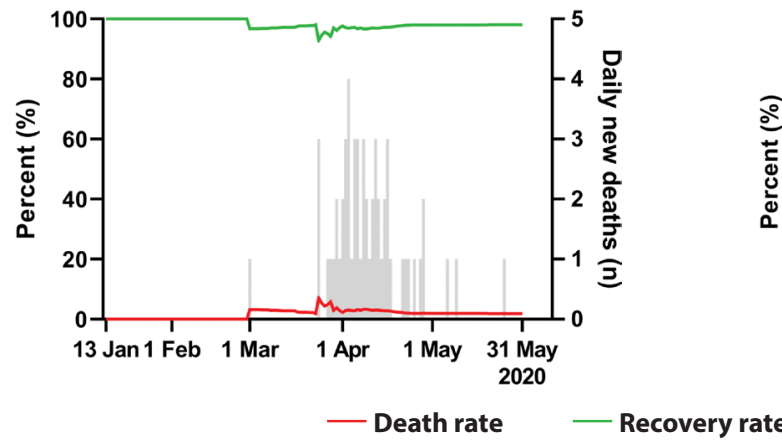

B

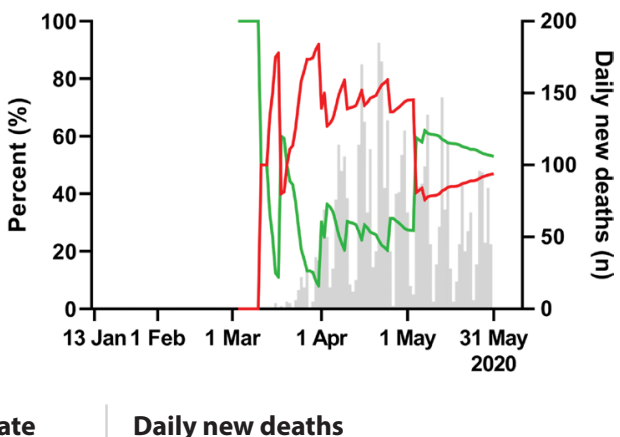

Figure 2. Outcomes of cases (recovery or death) among models.

The cumulative total deaths and recoveries were calculated from the cumulative number of closed cases. 
A

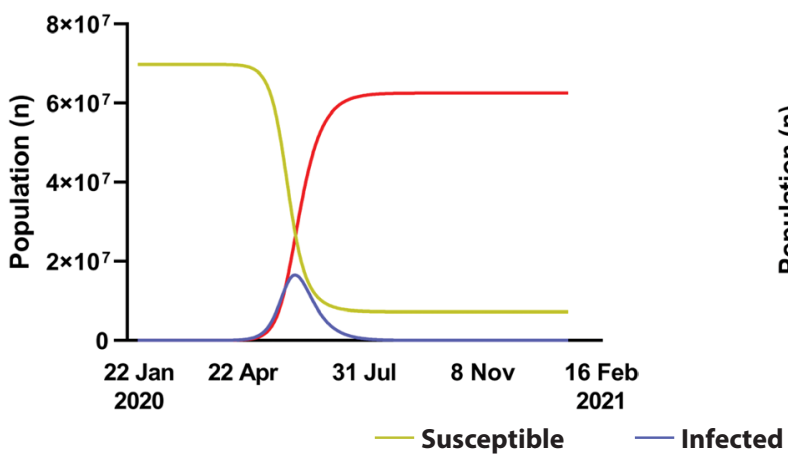

B

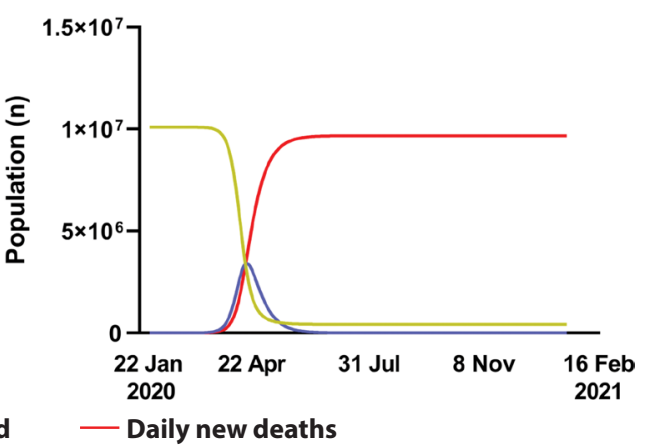

C

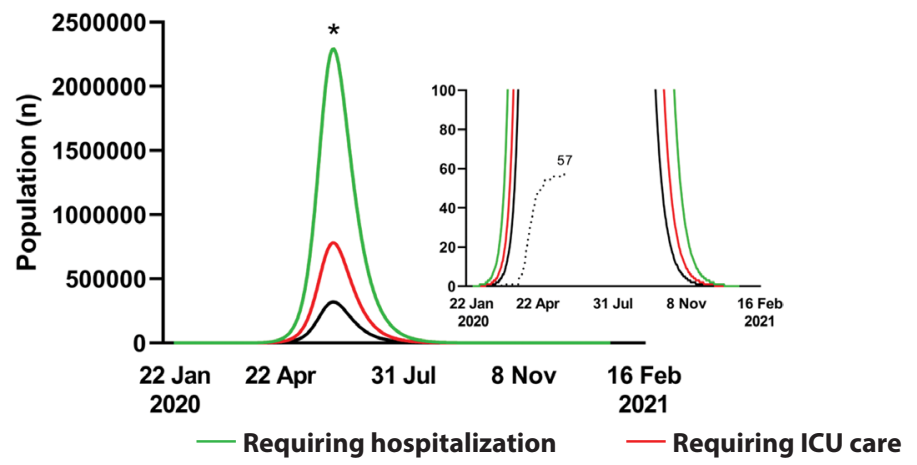

D

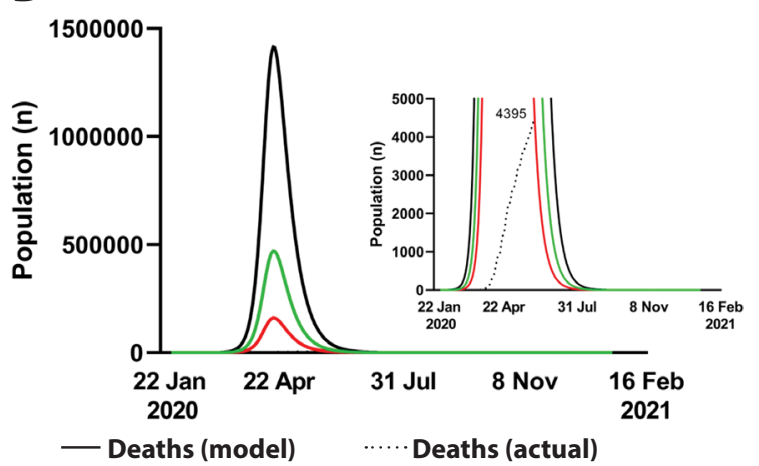

Figure 3. Prediction of COVID-19 between social restriction and herd immunity models.

The SIR model of COVID-19 for (A) Thailand and (B) Sweden. Prediction of the clinical outcome of COVID-19 in (C) Thailand and (D) Sweden, based on epidemiological data from China, is demonstrated. Additionally, $\left.{ }^{*}\right)$ indicates that the number of hospitalizations might exceed $16.5 \mathrm{M}$ persons, and the inset graph shows the cumulative actual deaths during the observation period.

Comparison of the clinical outcomes, mortality rate and crude death rate between models

A comparison of the clinical outcomes between models A and B, as of May 31, 2020, is shown in Figure 2. We found more confirmed COVID-19 cases and deaths in model B than in model A. In addition, the outcome of the COVID-19 pandemic was evaluated based on the mortality rate and crude death rate with regard to strategic control of COVID-19. Of note, model B in Sweden showed a higher mortality rate (11.7\%) and crude death rate (435 deaths per 1,000,000 population) than model $\mathrm{A}$ in Thailand (Table 1).

When the SIR model was used for predicting the COVID-19 status, Sweden (model B) showed the potential to end the COVID-19 epidemic before August 2020-which is earlier than the prediction for Thailand (model A) (268 vs. 368 days) (Figure 3A, 3B) - and the number of deaths would be over 1 million (Figure 3D). However, we found that both models projected much higher mortality than actual cases (Figure 3C, 3D). Interestingly, according to the SIR model, $34 \%$ of the Swedish population had already been infected by April 14, 2020 (Figure 3B).
Proposed parameter guidance on appropriate timing for mitigation of social restriction

We further studied parameters that would correlate with good outcomes after relaxation of social restriction. Interestingly, at least one log difference between total confirmed cases and active cases in the cubic spline interpolation with logarithm graph was demonstrated as a potential parameter for appropriate timing for mitigation of social restriction (Figure 4). As a proof of concept, the COVID-19 data for Hong Kong and Japan and the total world data collection were tested (Figure 4C-4E). While Hong Kong showed a comparable interpolation (1.17 log) difference between total confirmed cases and active cases with Thailand $(1.7 \log )$, Japan, which has developed a second wave of COVID-19 spread, reached only $>1 \log$ for a week (Figure 4D). Of note, as shown in Figure 4C, the value for Hong Kong shows a decreasing trend from $1.6 \log$ to $1.4 \log$ within the most recent week. Additionally, this assumption has been generalized and validated for other countries, which were classified as having been successful in COVID-19 control. ${ }^{26}$ Interestingly, as of May 15, 2020, all of the countries in the successful group demonstrated at least 1 log difference between total confirmed cases and active cases in the log curve between population (total confirmed and active cases) and time, and none of these countries showed a second wave of COVID-19 spread beyond this point (Figure 5). 
A

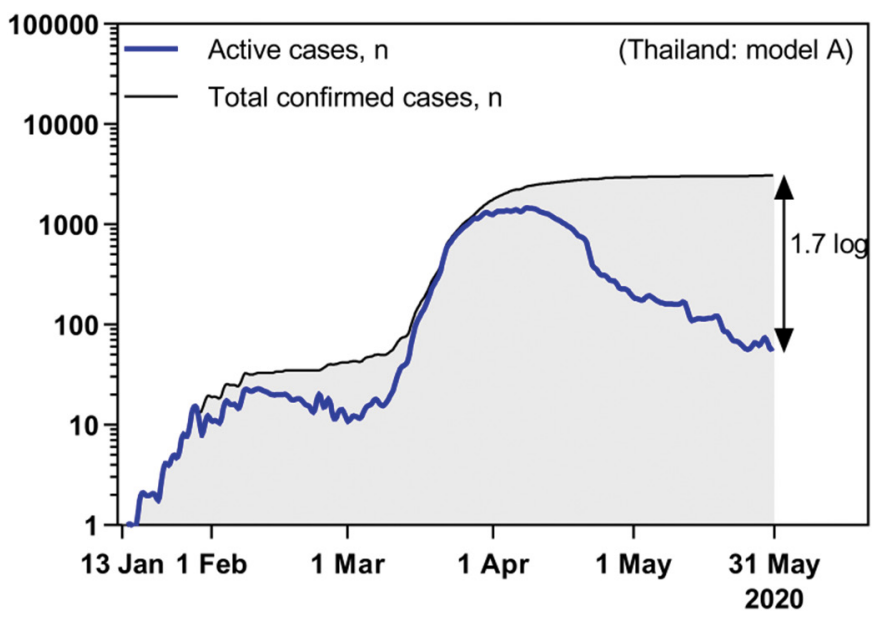

C

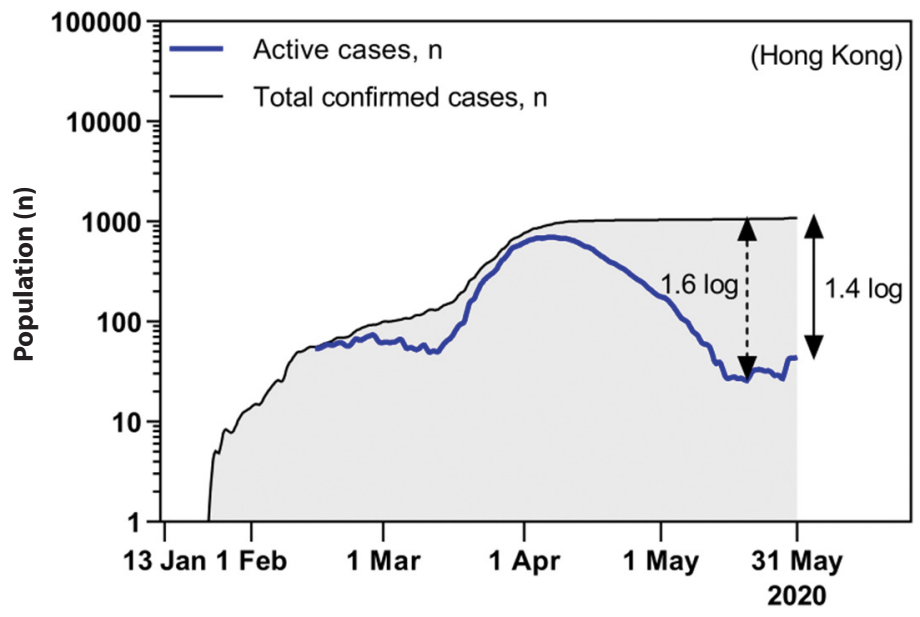

E

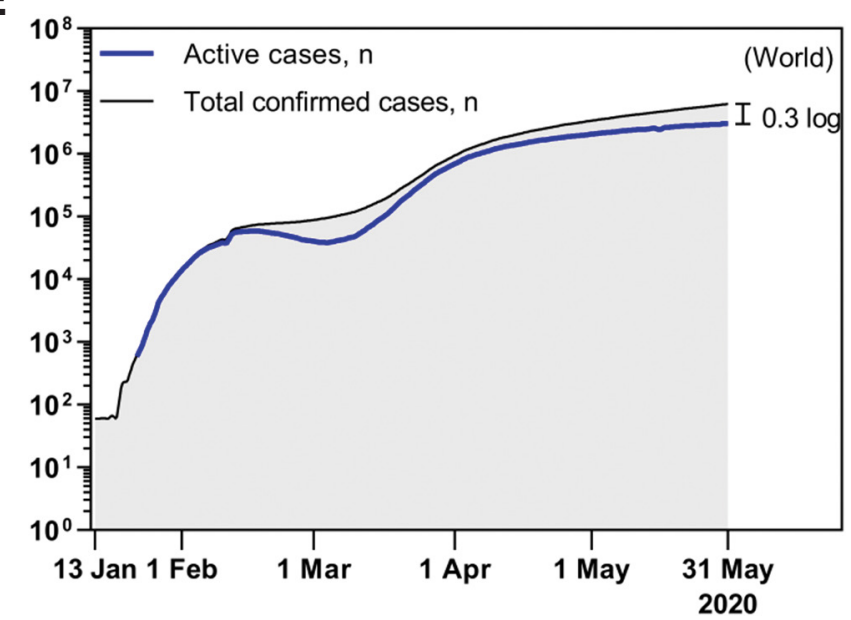

B

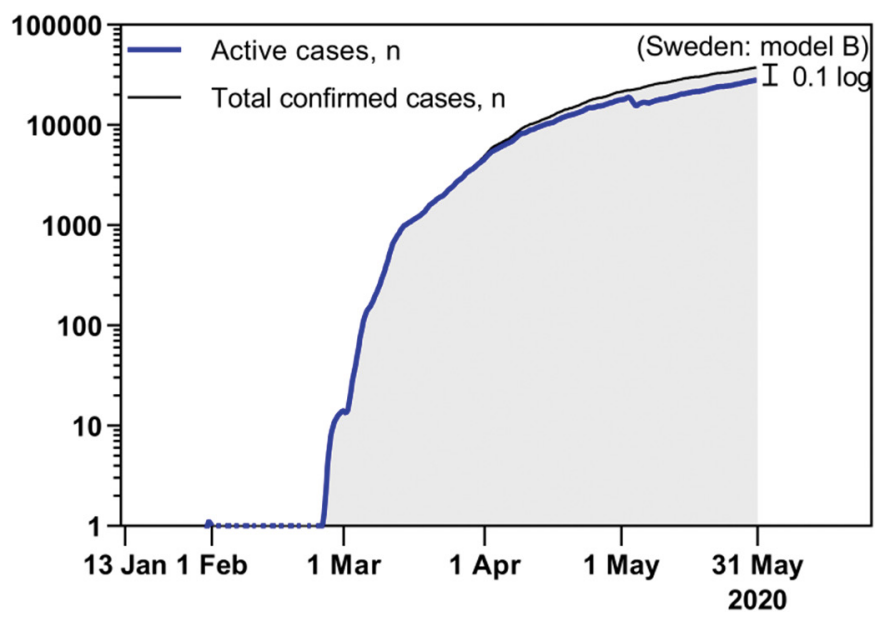

D

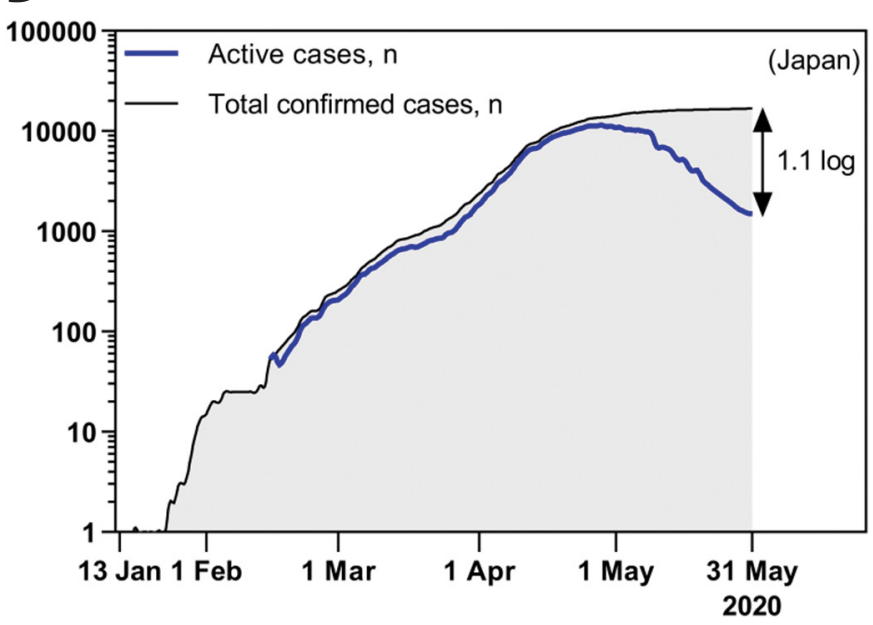

Figure 4. Cubic spline interpolation showing the comparison of the total confirmed cases and active cases between social restriction and herd immunity models for the COVID-19 pandemic.

The logarithmic curve distance parameter during the observation period of the COVID-19 status in (A) Thailand (model A), (B) Sweden (model B), (C) Hong Kong, and (D) Japan and (E) globally are demonstrated. While Japan increased the interpolation curve difference between the total confirmed cases and active cases $(1.1 \mathrm{log})$, Hong Kong tended to decrease the difference from $1.6 \log$ (dashed arrow) to $1.4 \log$ (solid line arrow) in a recent week (D). 
A

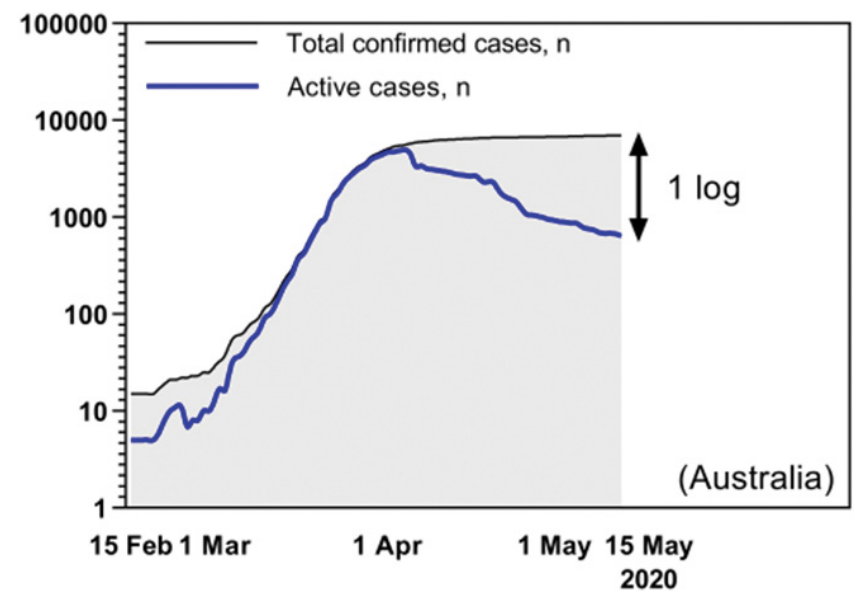

C

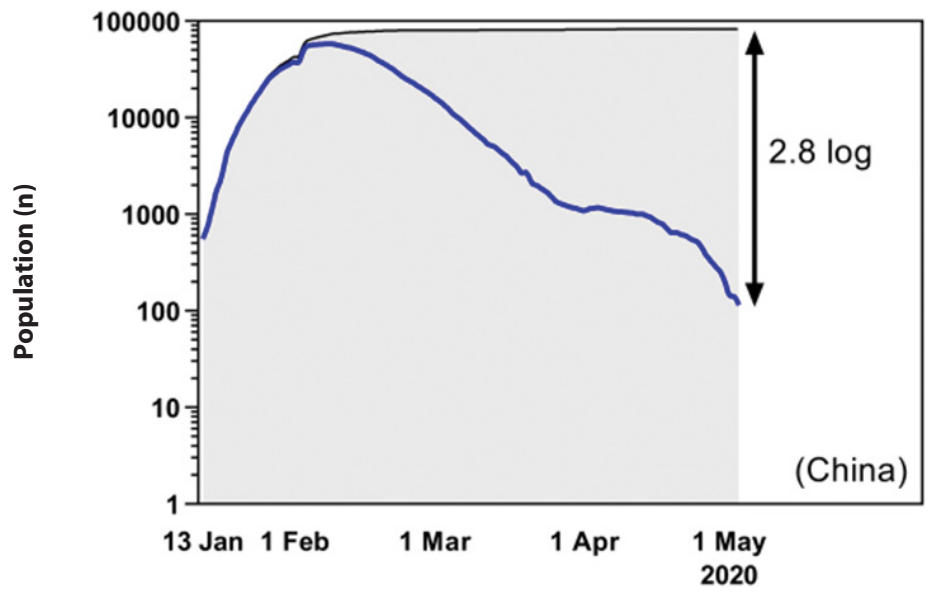

E

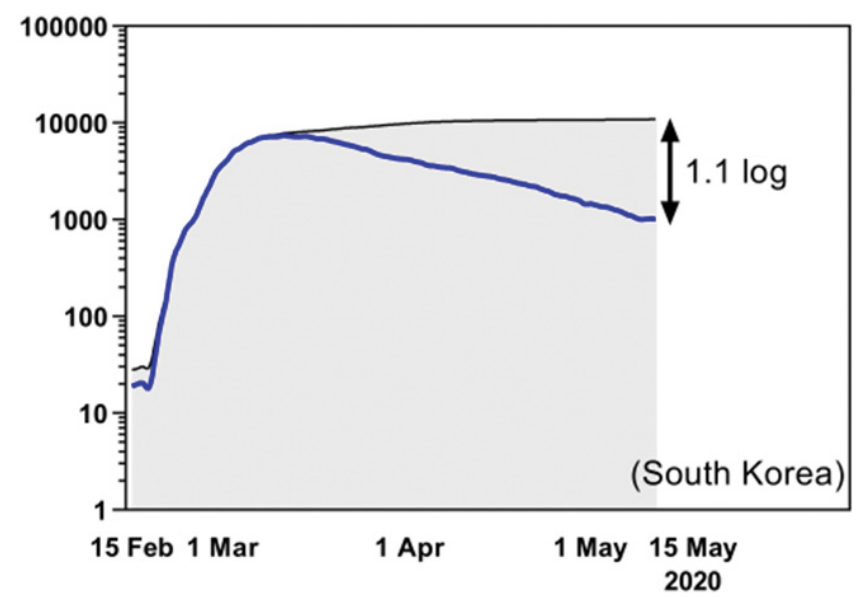

B

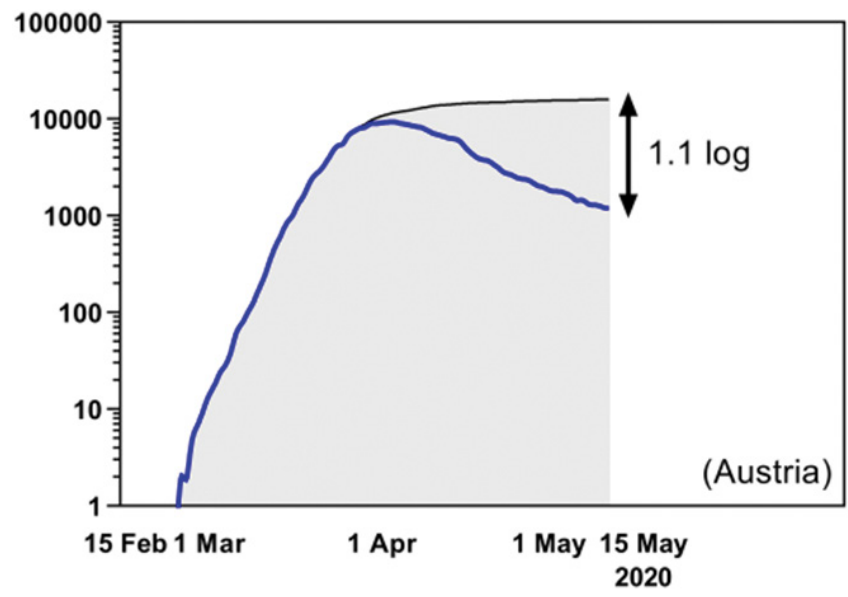

D

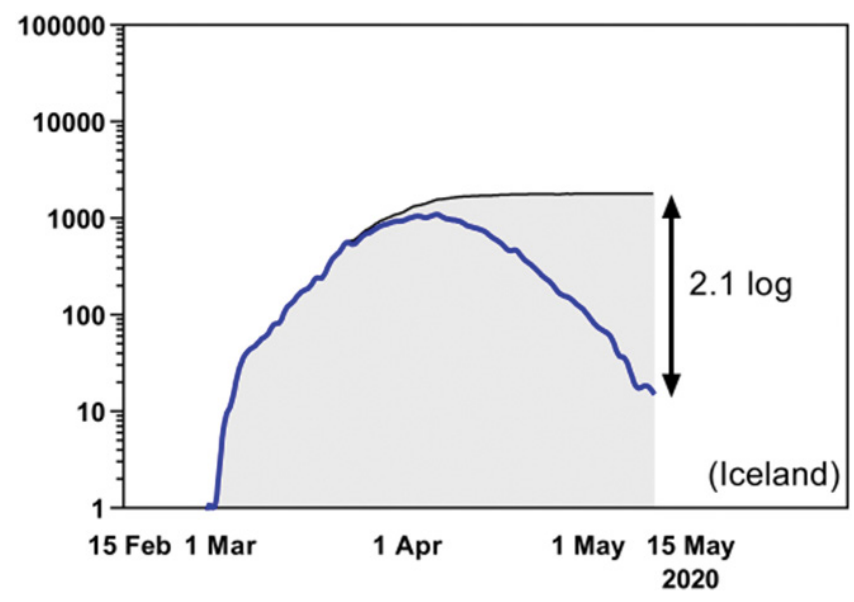

$\mathbf{F}$

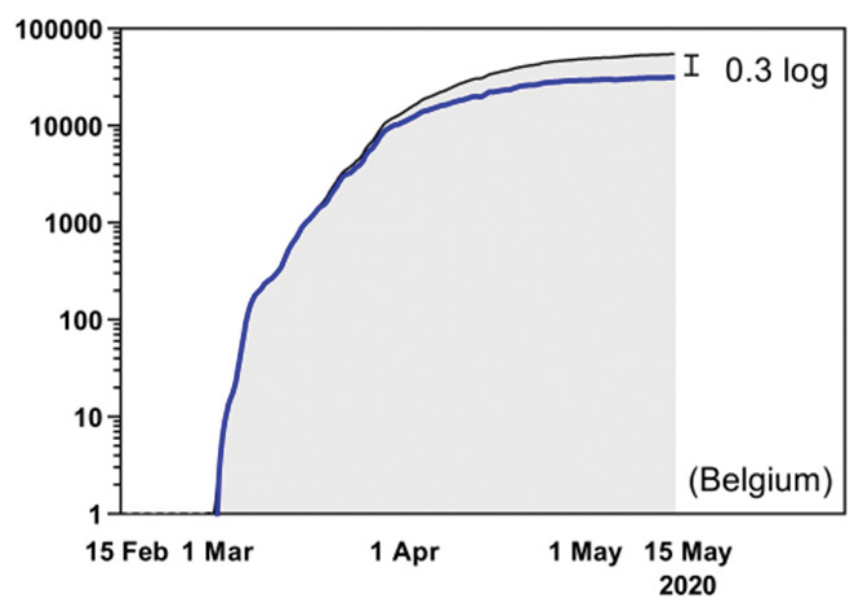

Figure 5. Cubic spline curve between the total confirmed cases and active cases in countries, classified according to the current COVID-19 status (as of May 15, 2020).

The difference between the total confirmed cases and active cases in (A-E) the countries classified as having a good COVID-19 status (> 1 log difference) and (F-J) fair COVID-19 status and (K-O) those needing urgent action $(<1$ log difference) are demonstrated. 
G
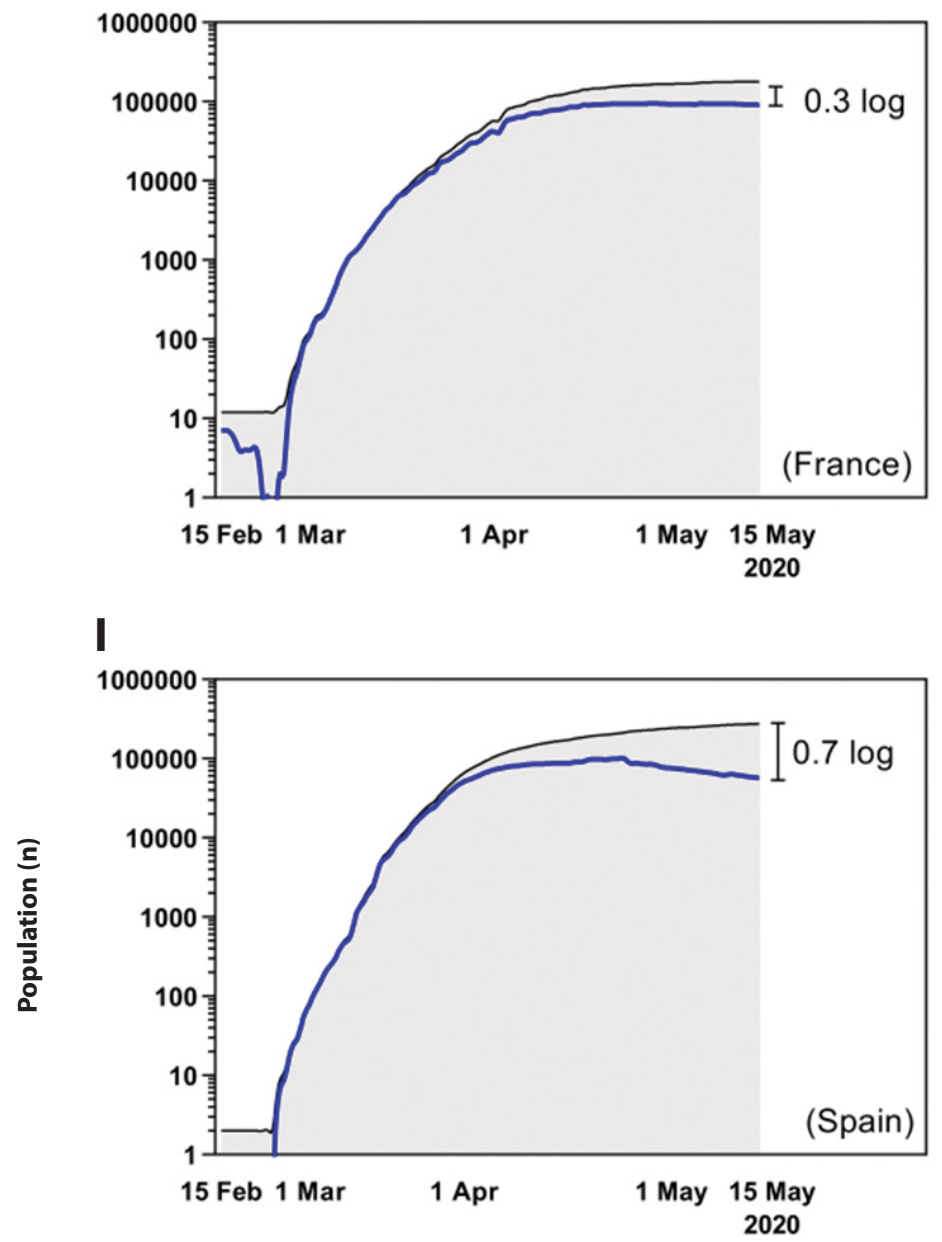

K

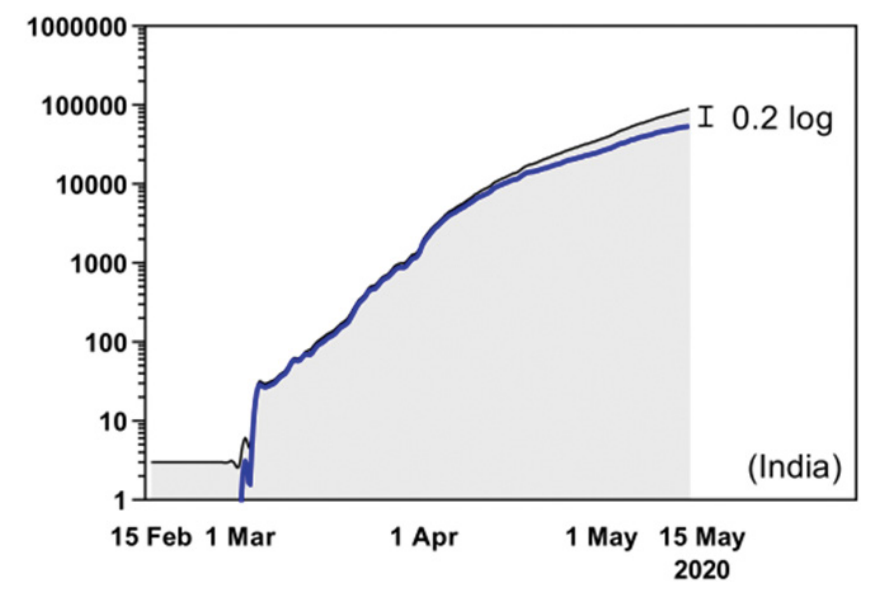

H

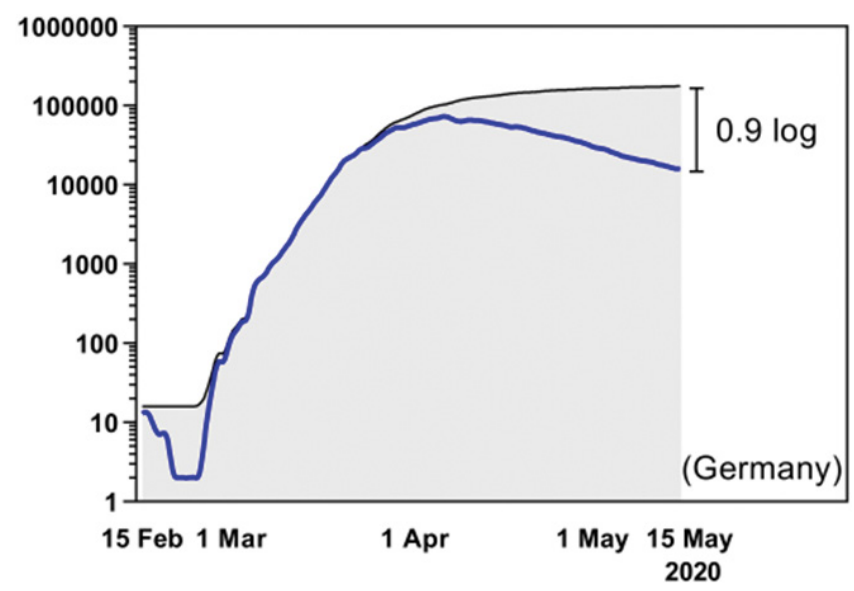

J

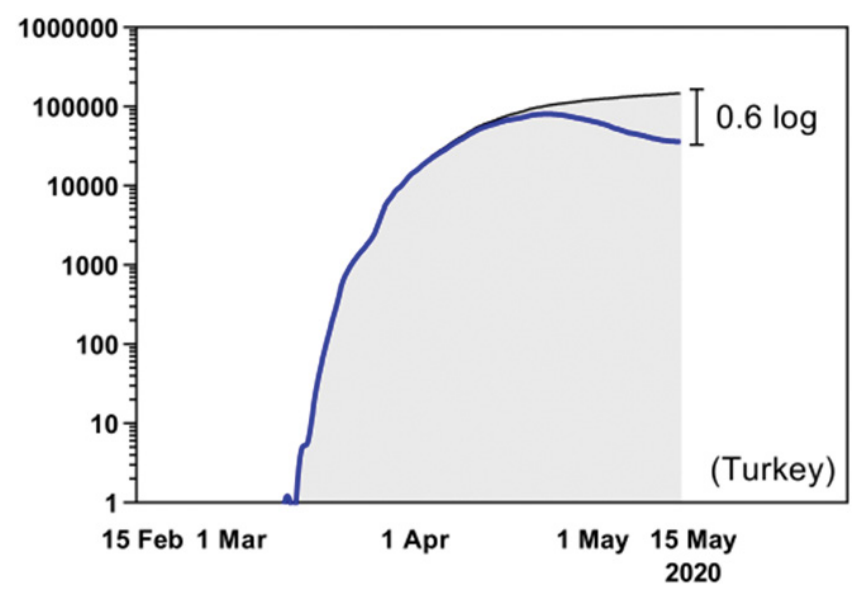

L

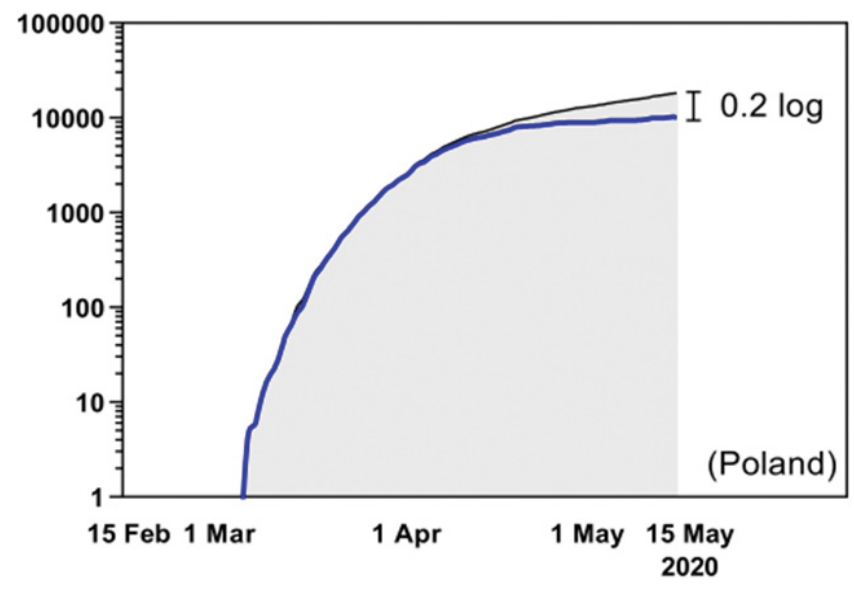

Figure 5. (Continued) 


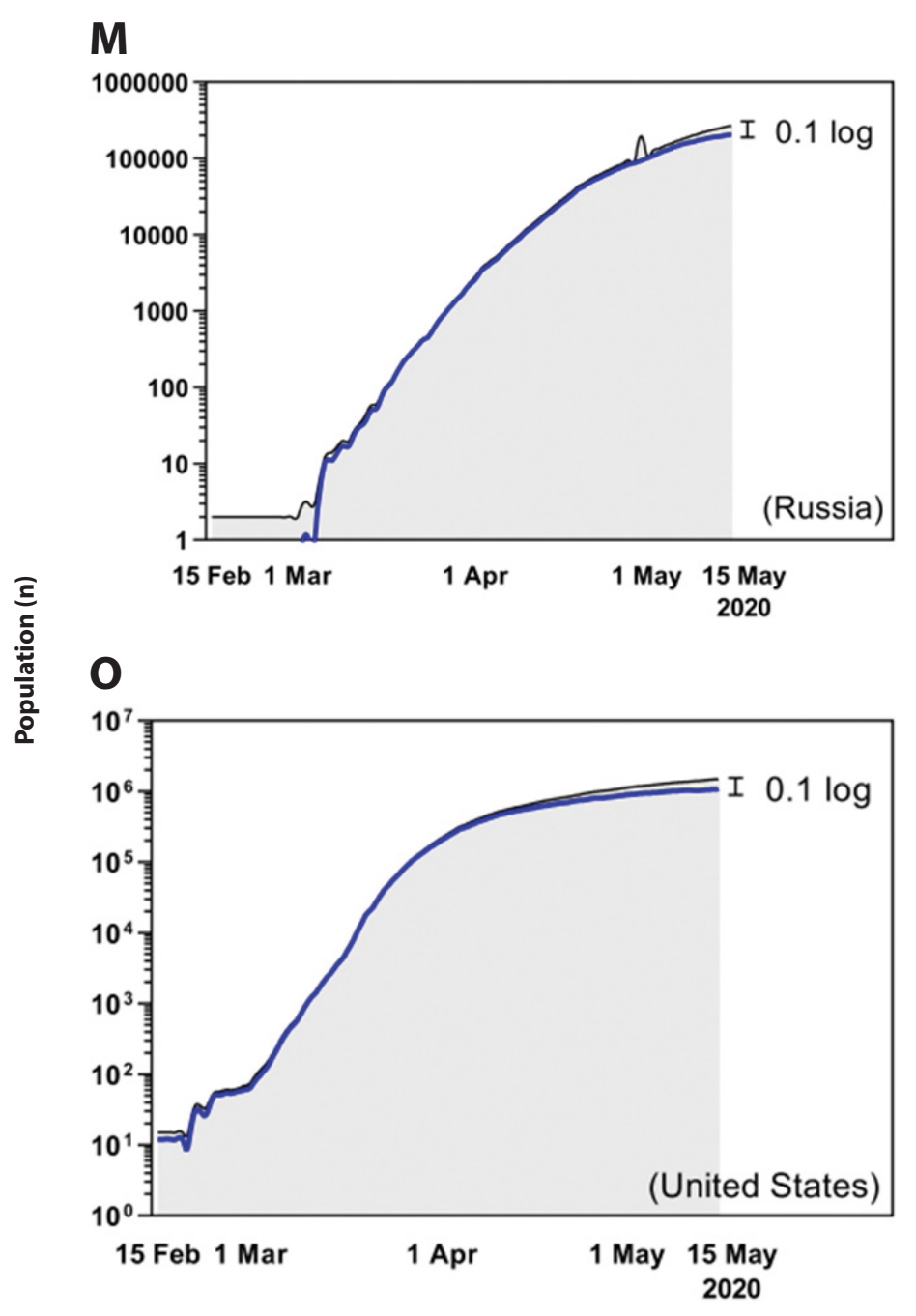

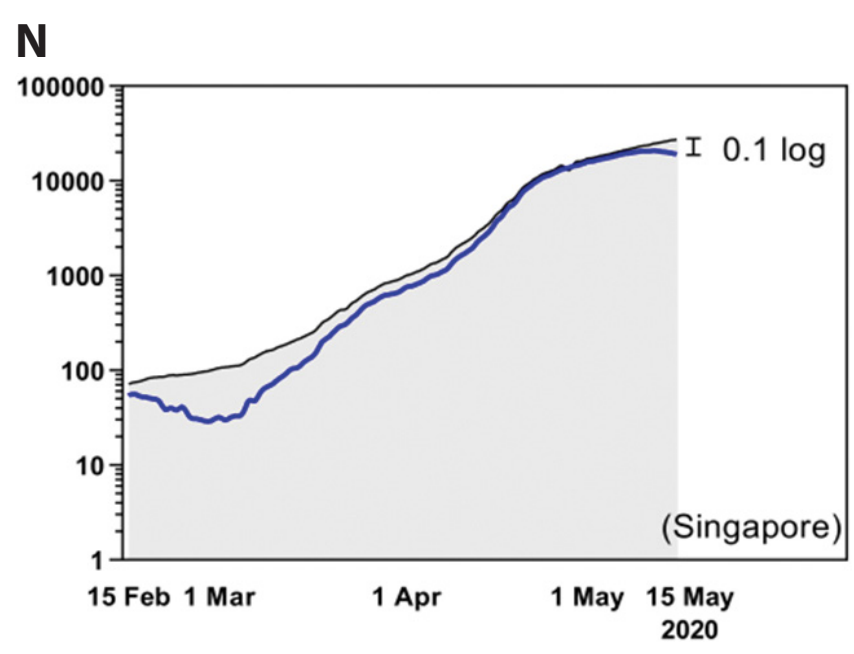

Figure 5. (Continued)

\section{Discussion}

This study explored COVID-19 control strategies, social restriction and herd immunity plans, and outcomes. Based on the SIR model analysis, the social restriction model needs an approximately three-month longer duration to control the disease than the herd immunity plan, despite the lower mortality rate associated with social restriction. In addition, the gap between the total confirmed cases and active cases is a potential indicator of the appropriate time to mitigate the social restriction policy.

While global scientists and the World Health Organization (WHO) are developing antiviral drugs and vaccines against COVID-19, the main strategy remains prevention processes. In the worst-case scenario, COVID-19 will likely remain for decades as an endemic disease, even with an effective vaccine, and a preventive policy will still be needed. Thailand has been expected to be a very high-risk country for SARS-CoV-2 spread because it is among the favorite tourist destinations for the Chinese, which is supported by a report of the first COVID-19 cases outside mainland China being detected in Bangkok. Despite intensive restriction control, the number of COVID-19 cases in Thailand reached 100 within less than 10 weeks (Figure 1A), but the maximum number of deaths per day was only 188 on March 22, 2020 (Figure 2). In contrast, it took 4 weeks for the cumulative confirmed cases in Sweden, with a herd immunity plan, to exceed that in Thailand, although the first case in Sweden was reported 2 weeks after the first Thai case (Figure 1A). However, the number of tests performed in Sweden was much higher than that in Thailand. Thailand reported a relatively low mortality rate compared to Sweden and a high recovery rate. The abrupt increase in the number of COVID-19 cases during the early phase in Sweden (Figures 1A and 2) might exceed the healthcare facilities and capacities, leading to the reported outcomes regardless of the impact on early implementation (Figure 1B).

However, country-specific cultures and norms could also contribute to COVID-19 outcomes. While the Thai people use the gesture 'Wai' for social communication, the traditional Western handshake may facilitate the spread of COVID-19. Regarding the wearing of face masks in public places from February 27 to May 4, 2020, most people in Thailand (71$90 \%$ ) consistently used face masks, whereas $<10 \%$ of the people in Sweden wore face masks. ${ }^{27}$ Additionally, the concern regarding COVID-19 among Thai people was high even before government policy implementation, possibly due to social media use. As shown in the timeline, approximately $90 \%$ of people living in the Bangkok metropolitan area wore a face mask 
outdoors from March 13, 2020, when a Thai celebrity in a Thai boxing match was diagnosed with COVID-19 infection (see Introduction). All of the earlier factors not only enhance the effectiveness of social restriction but also provide support for government policies. Despite the herd immunity plan (model B) in Sweden, the government also encouraged stay-at-home measures (particularly for the elderly), banned group activities, instated a social distancing policy, and closed academic institutes (high schools and universities). However, restaurants, bars, primary schools and most businesses and shops were still open. Perhaps the main differences between Thailand and Sweden were a strong mutual trust between public authorities and citizens and a health care system. Based on the data from April 22, 2020 from the Ministry of Health and Social Affairs, Government Office of Sweden, they have over 1,100 intensive care units for performing COVID-19-related tasks. ${ }^{28}$ Indeed, the study results showed some advantages of the social restriction model over the herd immunity model, although both models $\mathrm{A}$ and $\mathrm{B}$ showed positive effects on the actual mortality rate compared with the mortality rate predicted from SIR models. Furthermore, the social restriction model showed a lower death-to-hospitalization ratio compared with the herd immunity model (Figure 3C and 3D), with a trend showing a longer period for disease control (approximately 4 months) (Figure 3). In contrast to the SIR model, which demonstrated that over $34 \%$ of the population has been infected since April 14, 2020 (Figure 3B), the actual number of confirmed cases as of May 31, 2020 was only 37,542. This implies Sweden could take four to five years to reach full herd immunity, which requires $50-70 \%$ of the population to be exposed to SARS-CoV-2. However, the number of infected cases in Sweden remains unknown because only $2.2 \%$ of the population has been tested for COVID-19.

COVID-19 affects not only physical health but also the economy, unemployment, and mental health. Although model A has a lower mortality rate, this is not necessarily the most appropriate strategy. Furthermore, according to the herd immunity model, over $34 \%$ of Swedish people have already been infected SARS-Cov-2, which safeguards them from the second and third waves, which is different from the effect of the social restriction policy. Further study of viral seroprevalence between models based on several aspects (biophysical, mental, and socioeconomic health) is warranted.

The prediction parameter for the appropriate time for lifting lockdown was also studied based on both clinical judgment and an academic institutional report. ${ }^{26}$ At least one log difference between the total confirmed cases and active cases (almost equal to the cumulative number of total recovered cases) on the cubic spline interpolation could be a potential parameter for differentiating the countries with improving trends from others (Figures 4 and 5). Interestingly, Hong Kong had the highest difference $(0.5$ logs $)$ between the total confirmed cases and active cases in mid-March, as shown in Figure 4C; however, they could not maintain this status until early May, showing a > $1 \log$ difference in May 2020. On the other hand, those countries with $<1 \log$ difference on May 2020 (Figure 5) remains in difficult situation of COVID-19 as of early October 2020 .
Some limitations should be mentioned. First, the representative countries selected for the policy models (Thailand for social restriction and Sweden for the herd immunity plan) were based on the stringency index scores, which might not be accurate. It should be noted that the indices in this reference reported the number and strictness of government policy, but the scale was not linear, and a higher score did not necessarily mean a 'better' response than a lower score, so the qualitative assessment of the strategies in both countries is also important to consider. Second, the classic SIR (susceptible, infectious, recovered) model was excluded not only subsequently economic impacts but also asymptomatic carriers of SARS-CoV-2 in the community. The SIR model used here is only a simple one. As such, the predictions that come out might not be accurate enough, something that also depends on the published data. Nevertheless, our SIR model provides a theoretical framework to investigate the spread of SARSCoV-2 within two distinct social restriction policies. The model can give insights into the time evolution of the spread of the virus that the data alone does not. In this context, it could be as a benchmark for how efficiency of Covid-19 control is. The data from SIR model also, at least, provide the estimates of number of likely deaths in the future along with the declining trends in the number of Covid-19 infected individuals. Third, the clinical outcome model should be interpreted with cautions due to the epidemiology data was mainly based on China experience. Lastly, due to the continuation of the pandemic, our results may represent preliminary data. Further follow-up studies are warranted.

Taken together, the results show that a policy for COVID-19 control is essential for addressing this pandemic. However, it is difficult to determine which policy is too lenient or too stringent, as this depends on the individual context of each country. Social restriction is somewhat important for controlling mortality, while herd immunity may have the benefits of shortened infection durations and fewer concerns about reinfection. Moreover, the difference between total confirmed cases and active cases (more than one log) might be a good indicator of the outcome of COVID-19 control.

\section{Author contributions}

WC, AL, SC, and SK had roles in the study design, data collection, data analysis, data interpretation, and writing of the manuscript. WC, AL, KP, WP, PW, and PP had roles in the study design, data collection, data analysis, and data interpretation. All authors reviewed and approved the final version of the manuscript.

\section{Disclaimer}

The funding sources had no role in the study design, data collection, analysis, interpretation, or writing of the report.

\section{Financial support}

This study was partly supported by seed funding of the Division of Clinical Tropical Medicine, Faculty of Tropical Medicine, Mahidol University. 


\section{Potential conflicts of interests}

All authors declare no competing interests.

\section{References}

1. Cheng PK, Wong DA, Tong LK, Ip SM, Lo Ac, Lau CS, et al. Viral shedding patterns of coronavirus in patients with probable severe acute respiratory syndrome. Lancet. 2004; 363(9422): 1699-700.

2. Fine P, Eames K, Heymann DL. "Herd immunity": a rough guide. Clin Infect Dis. 2011; 52(7): 911-6.

3. Desai AN, Patel P. Stopping the Spread of COVID-19. JAMA. 2020; 323(15): 1516.

4. Desai AN, Aronoff DM. Masks and Coronavirus Disease 2019 (COVID-19). JAMA. 2020; 323(20): 2103.

5. Heesterbeek H, Anderson RM, Andreasen V, Bansal S, De Angelis D, Dye $\mathrm{C}$, et al. Modeling infectious disease dynamics in the complex landscape of global health. Science. 2015; 347(6227): aaa4339.

6. moph.go.th [Internet]. Nonthaburi: Department of Disease Control MoPHoT. Thailand Situation of COVID-19; c2020 [cited 2020 May 31]. Available from: https://ddc.moph.go.th/viralpneumonia/eng/index.php.

7. who.int [Internet]. Geneva: COVID-19 - WHO Thailand Situation Reports; c2020 [cited 2020 May 31]. Available from: https://www.who.int/thailand/ emergencies/novel-coronavirus-2019/situation-reports

8. Thomas H, Webster S, Petherick A, Phillips T, Kira B. Oxford COVID-19 Government Response Tracker [Internet]. Oxford: Blavatnik School of Government; 2020 [cited 2020 May 31]. Available from: https://www. bsg.ox.ac.uk/research/research-projects/coronavirus-government -response-tracker.

9. worldometer.info [Internet]. Delaware: COVID-19 Coronavirus Pandemic; c2020 [cited 2020 May 31]. Available from: https://www.worldometers. info/coronavirus/.

10. Han L, Nazri H, Seng TW, Him PC, Ligot D, Zaidon UH. COVID-19 Corona Tracker [Internet]. Malaysia; c2020 [cited 2020 May 31]. Available from: https://www.coronatracker.com/.

11. coronavirus.jhu.edu [Interent]. Baltimore: Johns Hopkins University CRC. COVID-19 Dashboard by the Center for Systems Science and Engineering (CSSE) at Johns Hopkins University (JHU). [cited 2020 May 31]. Available from: https://coronavirus.jhu.edu/map.html.

12. Hethcote HW. The Mathematics of Infectious Diseases. SIAM Review. 2000; 42(4): 599-653

13. Udomsamuthirun P CG, Tongkhonburi P,Meesubthong C. The reproductive index from SEIR model of Covid-19 epidemic in Asean. medRxiv. 2020: 2020.04.24.20078287.

14. Fernández-Villaverde J JC. Estimating and simulating a SIRD model of COVID-19 for many countries, states, and cities [Internet]. [cited 2020 May 23]. Available from: https://web.stanford.edu/ chadj/sird-paper.pdf.

15. Kucharski AJ, Russell TW, Diamond C, Liu Y, Edmunds J, Funk S, et al. Early dynamics of transmission and control of COVID-19: a mathematical modelling study. Lancet Infect Dis. 2020; 20(5): 553-8.
16. Bi Q, Wu Y, Mei S, Ye C, Zou X, Zhang Z, et al. Epidemiology and transmission of COVID-19 in 391 cases and 1286 of their close contacts in Shenzhen, China: a retrospective cohort study. Lancet Infect Dis. 2020; 20(8): 911-919.

17. Zhou F, Yu T, Du R, Fan G, Liu Y, Xinag J, et al. Clinical course and risk factors for mortality of adult inpatients with COVID-19 in Wuhan, China: a retrospective cohort study. Lancet. 2020; 395(10229): 1054-62.

18. who.org. [Internet]. Geneva: Report of the WHO-China Joint Mission on Coronavirus Disease 2019 (COVID-19). [cited 2020 Apr 30]. Available from: https://www.who.int/docs/default-source/coronaviruse/ who-china-joint-mission-on-covid-19-final-report.pdf.

19. Wu JT, Leung K, Leung GM. Nowcasting and forecasting the potential domestic and international spread of the 2019-nCoV outbreak originating in Wuhan, China: a modelling study. Lancet. 2020; 395(10225): 689-97.

20. Li Q, Guan X, Wu P, Wang X, Zhou L, Tong Y, et al. Early Transmission Dynamics in Wuhan, China, of Novel Coronavirus-Infected Pneumonia. N Engl J Med. 2020; 382(13): 1199-207.

21. worldometer.info [Internet]. Delaware: Population; c2020. [cited $2020 \mathrm{Apr}$ 30]. Available from: https://www.worldometers.info/world-population/.

22. Eubank S, Eckstrand I, Lewis B, Venkatramanan S, Marathe M, Barrett CL. Commentary on Ferguson, et al., "Impact of Non-pharmaceutical Interventions (NPIs) to Reduce COVID-19 Mortality and Healthcare Demand". Bull Math Biol. 2020; 82(4): 52.

23. Epdiemiological Working Gorup for NCIP Epidemic Response, Chinese Center for Disease Control and Prevention. The epidemiological characteristics of an outbreak of 2019 novel coronavirus diseases (COVID-19) in China. Zhonghua Liu Xing Bing Xue Za Zhi. 2020; 41(2): 145-51.

24. Govindarajulu US, Malloy EJ, Ganguli B, Spiegelman D, Eisen EA. The comparison of alternative smoothing methods for fitting non-linear exposure-response relationships with Cox models in a simulation study. Int J Biostat. 2009; 5(1): Article 2.

25. Alvarez MM, Gonzalez-Gonzalez E, Santiago GT. Modeling COVID-19 epidemics in an Excel spreadsheet: Democratizing the access to first-hand accurate predictions of epidemic outbreaks. medRxiv. 2020: 2020.03.23. 20041590.

26. endcoronavirus.org [internet]. Massachusetts: New England Complex Systems Institute Inc; c2020[cited 2020 May 31]. Who is beating COVID-19? Some are winning - some are not: 15 May; [about 18 screens]. Available from https://www.endcoronavirus.org/countries.

27. yougov.co.uk [Internet]. London: YouGov; c2018[cited 2020 May 28]. International COVID-19 tracker update: 18 May; [about 11 screens]. Available from: https://yougov.co.uk/topics/international/articles -reports/2020/05/18/international-covid-19-tracker-update-18-may.

28. government.se [Internet]. Stockholm: The Government Offices; c2020 [cited 2020 May 31]. Strategy in response to the COVID-19 pandemic: 6 Apr; [about 7 screens]. Available from:https://www.government.se/ articles/2020/04/strategy-in-response-to-the-covid-19-pandemic/.

\section{Supplemental data}

\section{Time Frame Spatial Distribution $<$ No. of confirmed cases, deaths $>$}

Jan 12, 2020 First country with a confirmed case outside mainland China; 61 years old Chinese female from Wuhan $<1,0>$

Jan 17, 2020 Second case in Thailand $<2,0>$

Jan 31, 2020 A case of a Thai taxi driver man with no travel history or contact with a confirmed case; he is the first Thai case $<19,0>$

Feb 15, 2020 First case of a health care worker $(\mathrm{HCW})<34,0>$

Feb 26, 2020 A case of a Thai man who lives in Don-Meung District. He concealed his travel history, there were many HCWs who came in contact with him $<40,0>$

Feb 29, 2020 First COVID-19-related death in Thailand $<42,1>$

Mar 6, 2020 Thai boxing match at Lumpini Stadium, 2,500 audience members $<48,1>$

Mar 8, $2020 \quad$ Illegal migrant who arrived from Korea to Thailand $<50,1>$

Mar 12, 2020 Eleven cases of COVID-19 from a nightclub $<72,1>$ 


\section{Supplemental data (Continued)}

\section{Time Frame Spatial Distribution < No. of confirmed cases, deaths $>$}

Mar 13, 2020 Thai celebrity (speaker at the Thai boxing match) is diagnosed with COVID-19 $<75,1>$

Mar 15, 2020 Cumulative number of patients exceeds $100<114,1>$

Mar 22, 2020 Bangkok starts closing shopping malls

Maximum number of new cases reached $\langle 599,1\rangle$

$[\mathrm{n}=+188]$

Mar 25, 2020 Thammasat Field Hospital (the first field hospital) opened $\langle 934,4\rangle$

Mar 26, 2020 Declaration of emergency situation along with cumulative cases exceeding $1,000<1,054,4\rangle$

Mar 31, 2020 Maximum daily deaths reached $\langle 1,651,10\rangle$

Apr 3, 2020 Thai Prime Minister announces 'curfew' nationwide (people were not allowed outdoor from 10 p.m. to 4 a.m.) and all airline services closed $<1,978,19>$

Apr 4, $2020 \quad$ Cumulative cases exceed $2,000<2,067,20>$

Apr 27, 2020 First day of 1 -digit increase ( $<10$ cases/day) in new confirmed cases $<2,931,52>$

May 1, 2020 Domestic airline reopened with physical distancing restrictions $<2,960,54>$

May 3, 2020 First phase of easing of the COVID-19 lockdown: BMA allowed to partially reopen Bangkok and relax physical distancing from $2 \mathrm{~m}$ to $1.5 \mathrm{~m}$ in some facilities, including food courts and supermarkets $<2,969,54>$

May 9, $2020 \quad$ Cumulative cases exceed 3,000<3,004, 56>

May 18, 2020 Second phase of easing of restrictions $\langle 3,031,56\rangle$

June 1, 2020 Third phase of easing of restrictions $<3,082,57>$ 